Check for updates

Cite this: RSC Adv., 2017, 7, 31333

Received 15th May 2017

Accepted 13th June 2017

DOI: $10.1039 / \mathrm{c} 7 \mathrm{ra05463g}$

rsc.li/rsc-advances

\section{Unusual renormalization group (RG) flow and temperature-dependent phase transition in strongly-insulating monolayer epitaxial graphene $\uparrow$}

\author{
Lung-I. Huang, ${ }^{\text {ab }}$ Yanfei Yang, ${ }^{\text {ac }}$ Chieh-Wen Liu, ${ }^{\text {ad }}$ Randolph E. Elmquist, (D) ${ }^{a}$ \\ Shun-Tsung Lo, *d Fan-Hung Liu ${ }^{d}$ and Chi-Te Liang (iD *bde
}

\begin{abstract}
By changing the measurement temperature ( $T$ ), one can vary the effective sample size so as to study the renormalization group (RG) (or $T$-driven) flow of a semiconductor, a topological insulator, or a graphene device in the complex conductivity plane. Here we report RG flow of large-area, strongly disordered monolayer graphene epitaxially grown on SiC, which becomes insulating as $T$ decreases for zero magnetic field. We observe cusp-like RG flow towards $\left(\sigma_{x y}=e^{2} / h, \sigma_{x x}=e^{2} / h\right)$ where $\sigma_{x y}$ and $\sigma_{x x}$ are Hall conductivity and diagonal conductivity respectively. Such features, indicative of a fixed-temperature phase transition, have never been observed before and cannot be explained by existing RG models based on a modular symmetry group. Therefore, our results suggest the need for new theoretical models and experimental study leading to an understanding of strongly disordered two-dimensional materials such as graphene, few-layer black phosphorus, WSe ${ }_{2}$, and so on.
\end{abstract}

\section{Introduction}

Graphene, which is a single layer of carbon atoms bonded in a honeycomb lattice, ${ }^{\mathbf{1 , 2}}$ has continued to attract worldwide interest. Graphene prepared by mechanical exfoliation ${ }^{3}$ is of ultra-high quality and thus allows one to probe fascinating physical phenomena such as the Berry phase, ${ }^{\mathbf{1 , 2}}$ anomalous integer quantum Hall effects, ${ }^{\mathbf{1 , 2}}$ fractional quantum Hall effects, ${ }^{4-7}$ imaging cyclotron orbits, ${ }^{8}$ and so on. However, its limited size may not be suitable for maintaining the quantum Hall resistance (QHR) standard, which provides the unit of resistance in the International System (SI), or for large-scale integration of electronic devices. In contrast, monolayer graphene epitaxially grown on $\mathrm{SiC},{ }^{9}$ which can be of wafer-size and most importantly does not require any transfer process, is now widely believed to be an ideal candidate for replacing existing GaAs-based QHR reference standards. ${ }^{10-16}$ In addition, recently it has been shown that monolayer epitaxial graphene grown on SiC is an interesting platform for studying the insulatorrelativistic quantum Hall transition in graphene, ${ }^{17,18}$

${ }^{a}$ National Institute of Standards and Technology (NIST), Gaithersburg, MD 20899, USA. E-mail: shuntsunglo@mail.ncku.edu.tw

${ }^{b}$ Department of Physics, National Taiwan University, Taipei 106, Taiwan. E-mail: ctliang@phys.ntu.edu.tw

'Joint Quantum Institute, University of Maryland, College Park, MD 20742, USA ${ }^{d}$ Graduate Institute of Applied Physics, National Taiwan University, Taipei 106, Taiwan

${ }^{e}$ Department of Physics, Stanford University, Stanford, CA 94305, USA

$\dagger$ Electronic supplementary information (ESI) available. See DOI: $10.1039 / \mathrm{c} 7 \mathrm{ra05463g}$ insulating behavior, ${ }^{17-19}$ variable range hopping, ${ }^{19}$ and so forth. Such measurements may well reveal the true ground-state of disordered graphene and shed light on possible band-gap opening in graphene on SiC. ${ }^{20}$

Pioneering work shows that by changing the measurement temperature $T$, one can effectively vary the size of a sample and study the temperature-driven (renormalization group (RG)) flow of the device. ${ }^{\mathbf{2 1 , 2 2}}$ Such a RG flow diagram is extremely useful for understanding the quantum Hall $(\mathrm{QH})$ effect and the $\mathrm{QH}$ plateau-plateau transition (normally based on the semicircle law ${ }^{21-25}$ ) which is a quantum phase transition. Let us consider the simplest case. For a system with a single conduction channel the semicircle represents a critical boundary for the $\nu=$ $2 \mathrm{QH}$ state, with a stable point on the $x$-axis at $\left(\sigma_{x y}, \sigma_{x x}\right)=\left(2 e^{2} / h\right.$, $0)$. An unstable point ${ }^{18}$ at $\left(\sigma_{x y}=e^{2} / h, \sigma_{x x}=e^{2} / h\right)$ represents the boundary between two types of RG flow. To the left of the unstable point, the RG flows are towards $\left(\sigma_{x y}=0, \sigma_{x x}=0\right)$ which is the insulating state. To the right of the unstable point, the RG flows are towards $\left(\sigma_{x y}=2 e^{2} / h, \sigma_{x x}=0\right)$, which is the $\nu=2 \mathrm{QH}$ state (Fig. 1).

RG flow lines in disordered two-dimensional (2D) systems are understood by referring to the semicircle law with a clear unstable point. Such results provide important information on the insulating behaviour in monolayer epitaxial graphene grown on $\mathrm{SiC}^{17-19}$ and the transition ${ }^{17,18}$ at low fields between an insulator and the $\nu=2 \mathrm{QH}$ state, which cannot be seen in many other graphene-like systems. For example, in our previous work, RG flow approximated by the semicircle law is only observed in the strongest disorder device even if clear crossing points in the longitudinal resistivity $\rho_{x x}$ are observed in all three monolayer 


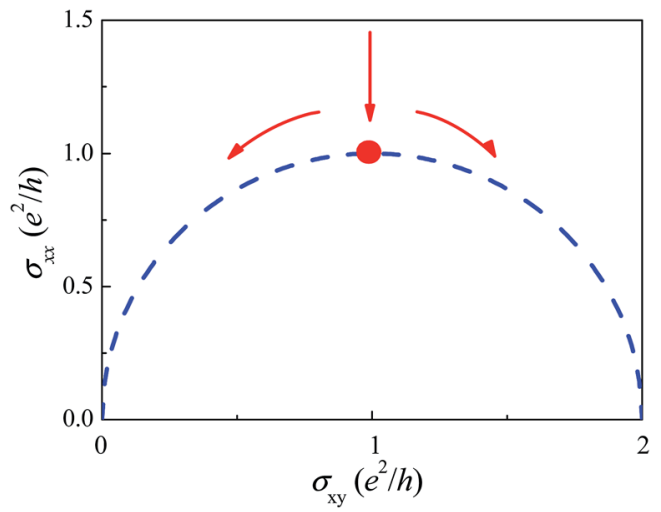

Fig. 1 Schematic diagram showing RG flow in the conductivity plane. The red dot corresponds to the unstable point. The red arrows indicate the directions of the RG flow.

graphene devices grown on SiC. ${ }^{18}$ Such results suggest that the unique insulator-relativistic $\mathrm{QH}$ transition in disordered graphene $^{17}$ is better studied by the RG flows rather than by the crossing point in $\rho_{x x}$ since RG flows described by the semicircle law strongly point to the floating-up of the $N=0$ Landau level at low magnetic fields. Moreover, the unstable fixed point in the RG flows unequivocally shows signature of a quantum phase transition. In order to further probe the RG flow in graphene and possibly new physical phenomena related to the unique relativistic $\nu=2 \mathrm{QH}$ state, we measure a disordered graphene device on $\mathrm{SiC}$ that is strongly insulating in the sense that the magneto-resistance decreases with increasing temperature $(T \leq$ $100 \mathrm{~K}$ ) over a wide range of temperature and magnetic field. Such a device is more disordered compared with those studied $^{17,18}$ in the sense that the zero-field resistivity is well above the quantum resistance $h / e^{2}$. Interestingly, we observe cusp-like RG flow towards $\left(\sigma_{x y}=e^{2} / h, \sigma_{x x}=e^{2} / h\right)$ which cannot be explained by existing RG models mostly based on the modular symmetry group. Our new experimental results suggest a fixed-temperature phase transition instead of a magnetic-field-induced quantum phase transition in strongly insulating graphene. We suggest that further theoretical and experimental work is required for understanding this hitherto unexplored research area.

\section{Results and discussion}

Fig. 2 shows the longitudinal and Hall resistivities of the device at different temperatures. At $T=15 \mathrm{~K}$, the carrier density and mobility of the device are calculated to be $9 \times 10^{10} \mathrm{~cm}^{-2}$ and $830 \mathrm{~cm}^{2} \mathrm{~V}^{-1} \mathrm{~s}^{-1}$. At $T<15 \mathrm{~K}$, strongly insulating behaviour in $\rho_{x x}^{\prime}$ makes the symmetrized $\rho_{x x}$ and $\rho_{x y}$ unreliable therefore we concentrate on data for $T \geq 15 \mathrm{~K}$. At zero magnetic field, the sample behaves as an insulator in the sense that $\rho_{x x}$ always decreases with increasing temperature. ${ }^{26}$ For $T \leq 100 \mathrm{~K}, \rho_{x x}(B)$ decreases with increasing $T$ over a wide range of $B(\leq 4.5 \mathrm{~T}){ }^{27}$

Fig. 3(a) and (b) show the converted $\sigma_{x x}$ and $\sigma_{x y}$ at different temperatures. Here, the insulating nature of the device is clearly observed from the increase in $\sigma_{x x}$ with $T$ for all values of $B$

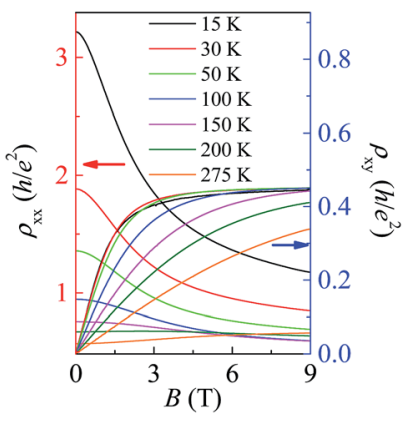

Fig. 2 Longitudinal and Hall resistivities $\left(\rho_{x x}\right.$ and $\left.\rho_{x y}\right)$ at various temperatures $T$, with temperature denoted by the color of the line.

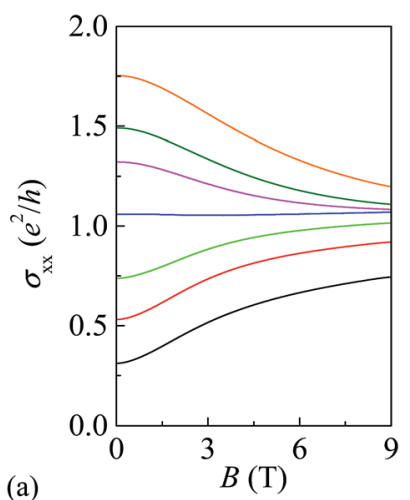

(a)

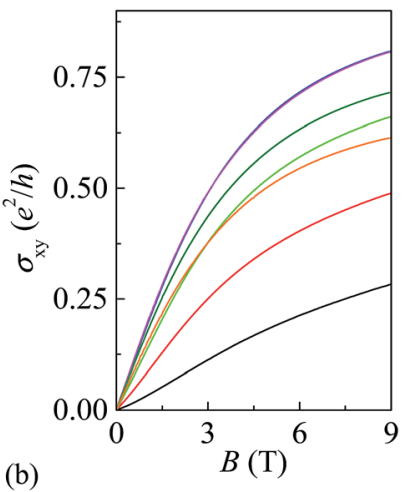

Fig. 3 (a) $\sigma_{x x}$ (b) $\sigma_{x y}$ as a function of $B$ at various $T$. The line colors correspond to temperatures as given in Fig. 2.

studied, while $\sigma_{x y}$ displays maximal values at $T \approx 100 \mathrm{~K}$ for all $B$. The diagonal conductivity $\sigma_{x x}$ is almost magnetic-fieldindependent for $T \approx 100-150 \mathrm{~K}$. Such an observed temperature dependence shows that even though signs of a quantum Hall-like step close to $h /\left(2 e^{2}\right)$ in $\rho_{x y}$ are seen at low temperature, we do not have a true $\nu=2 \mathrm{QH}$ state. A possible reason is that the disorder within the sample is so strong that the $\mathrm{QH}$ state is destroyed. That $\sigma_{x y}$ does not show quantized plateau values close to $2 e^{2} / h$ is due in part to large $\rho_{x x}$ in the high-field regime (Fig. 3(b)). Such results again suggest that the device does not enter the $\nu=2 \mathrm{QH}$ state.

We next develop the RG flow diagram of $\left(\sigma_{x y}, \sigma_{x x}\right)$ as shown by superimposed arrows pointing in the direction of the lowest $T$ at a series of fixed $B$ (Fig. 4(a) and (b)). For low $B$ the $T$-driven 

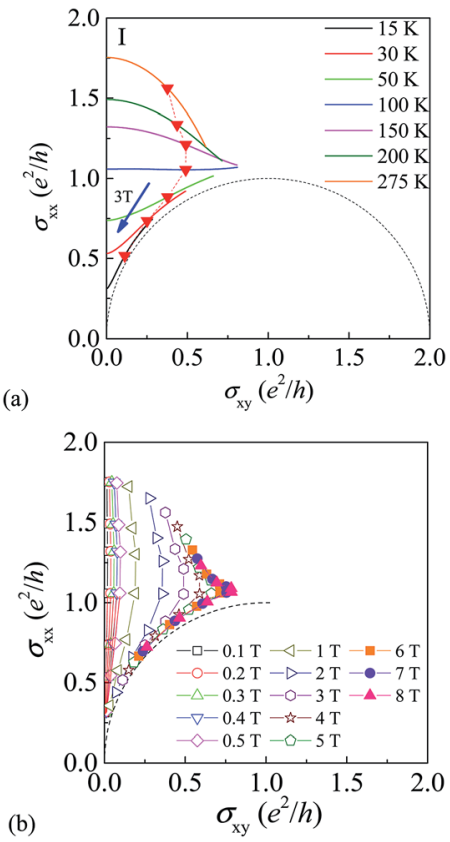

Fig. 4 (a) Conductivity $\sigma_{x x}$ plotted against $\sigma_{x y}$ EG4. The dotted curves denote the theoretical prediction of semicircle $\sigma_{x y}-\sigma_{x x}$ relation for the 0-2 transition. Each group of triangle markers connected by dashed lines denotes the data for the same magnetic field $(B=3 \mathrm{~T})$. The arrows indicate the flow line to the low temperature extreme at fixed magnetic fields. (b) Detailed RG flow over a wide range of $T$.

flow forms a set of curves that converge toward the insulating state at $(0,0)$. Such results strongly demonstrate that the device behaves as an insulator. Empirically, we observe quadratic decrease in $\rho_{x x}(B)$ and linear rise in $\rho_{x y}(B)$ over a wide temperature range for $B<0.3 \mathrm{~T}$, where the flow curves follow nearly straight paths along the $\sigma_{x x}$ axis. The quadratic negative MR is indicative of strong localization of carriers. ${ }^{28}$ For $B>0.3 \mathrm{~T}$, the curves for fixed $B$ bend outward in the range near $\sigma_{x x}=e^{2} / h$ and at higher $B$ a cusp appears near the unstable point at $\left(e^{2} / h, e^{2} / h\right)$. We wish to point out that such cusp-like RG flow cannot be observed in a similar GaAs-based disordered electron system in which the device is in the insulating regime with a $\nu=2 \mathrm{QH}$-like plateau structure (ESI Fig. $2 \dagger$ ). Such results strongly suggest that the cusp-like RG flow is a unique feature in strongly disordered graphene.

Quantum phase transitions (QPTs) in the QH regime may lie between adjacent $\mathrm{QH}$ states or separate the $\mathrm{QH}$ state from insulating states at either low or high perpendicular $B$ fields in two dimensions. ${ }^{29-32}$ The cusp in RG flow does not resemble a QPT, but rather suggests a fixed-temperature phase transition, as shown in Fig. 5(a), with a field-independent crossing at $\sigma_{x x} \approx$ $e^{2} / h$. This high-disorder state displays features of the $\nu=2 \mathrm{QH}$ state by approaching $\rho_{x y} \approx h /\left(2 e^{2}\right)$ at high fields and low $T$. Meanwhile, $\sigma_{x x} \approx e^{2} / h$ near the field-independent crossing and continues to rise smoothly as $T$ increases, resulting in the crossover from positive to negative slope of the diagonal magneto-conductivity $v s$. $T$ as shown in Fig. 4(a). To further illustrate this interesting behavior, we expand the plot of $\rho_{x x}(B)$ at high $T$ in Fig. 5(b) and find that there is a crossover from

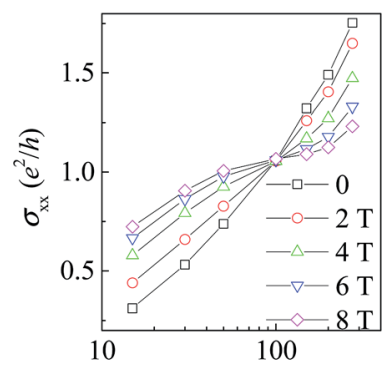

(a)

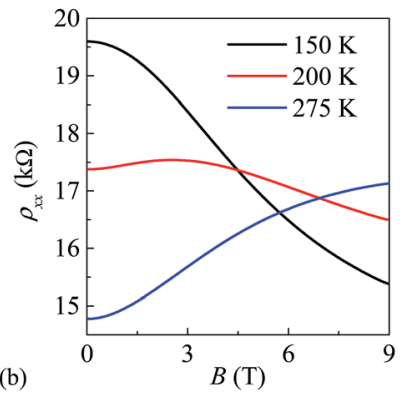

Fig. 5 (a) Temperature dependence of the diagonal conductivity for device EG4 in the range $15 \mathrm{~K} \leq T \leq 275 \mathrm{~K}$. The crossing point occurs at $T=(97 \pm 1) K$. (b) $\rho_{x x}(B)$ in the high- $T$ regime.

negative to positive MR with increasing $T$. Jabakhanji et al. ${ }^{33}$ observed positive magnetoresistance (MR) in highly doped graphene grown by chemical vapor deposition on $\mathrm{SiC}$ and argued that it is due to the energy dependence of mobility that is prominent at high $T$. This dependence may result from scattering by ionized impurities or by phonons produced at the graphene/SiC interface. In their work at low $T$ the $T$-independent crossing point in $\rho_{x x}$ (superimposed on the background parabolic negative MR) was observed when the product of mobility and magnetic field is equal to 1 and is presumably due to electron-electron interaction corrections. Moreover, with increasing $B$ above the $T$-independent crossing point, Landau quantization gave rise to Shubnikov-de Haas ( $\mathrm{SdH}$ ) oscillations in $\rho_{x x}$. In our experiments, the sample shows neither $\mathrm{SdH}$ oscillations nor the well-developed $\mathrm{QH}$ effect, probably due to stronger disorder within this graphene device. The crossover from negative MR to positive MR may be due to strong electronphonon scattering which increases the high-field MR.

In conclusion, we observe an insulating state that persists at all fields studied and coexists with weak QH-like characteristics at moderate and higher field strength. For $T<100 \mathrm{~K}, \sigma_{x x}$ shows monotonic decrease with increasing $B$. In contrast, For $T>100 \mathrm{~K}$ $\sigma_{x x}$ increases with increasing $B$, and for $T \approx 100 \mathrm{~K}$ it passes through a field-independent crossing. In the low field insulating regime, carriers are localized as a result of the presence of strong disorder, and display quadratic negative MR. At higher $B$ a cusp appears near the unstable point at $\left(e^{2} / h, e^{2} / h\right)$ in the RG flow diagram. A possible reason is that the $\nu=2 \mathrm{QH}$ state in graphene on $\mathrm{SiC}$ is very robust so that even strong disorder and high $\rho_{x x}$ within the device can co-exist with the presence of a QH-plateau-like structure in $\rho_{x y}$. We speculate that this may lead to the observed cusp-like RG flow towards $\left(\sigma_{x y}=e^{2} / h, \sigma_{x x}=\right.$ $\left.e^{2} / h\right)$. However, as our new results cannot be explained by any 
existing RG models based on the modular symmetry group, further experimental and theoretical studies are needed in order to probe this hitherto unexplored interesting area in the field of disordered 2D materials. ${ }^{34}$

\section{Experimental}

\section{Materials preparation}

During the high-temperature annealing process near $T=$ $1900{ }^{\circ} \mathrm{C}$, Si atoms sublimate and C atoms reorganize into graphene layers while the $\mathrm{SiC}(0001)$ substrate forms atomically-flat terraces. The initial graphene layer lies on top of and across the terraces, forming a $(6 \sqrt{ } 3 \times 6 \sqrt{ } 3) R 30^{\circ}$ reconstruction on the $\mathrm{Si}$ face that is azimuthally oriented to the $\mathrm{SiC}$ lattice..$^{35}$ This zerolayer graphene is covalently bonded to $\mathrm{Si}$ atoms in the substrate and is non-conducting. It is often described as the buffer layer on the Si-face $\mathrm{SiC}(0001)$ surface because of its effect on the carrier concentration in conducting graphene layers. Conducting epitaxial graphene is created as additional carbon forms a new buffer layer interface beneath an electrically-active monolayer of graphene. ${ }^{36}$

\section{Device fabrication}

Hall bar devices of width $w=100 \mu \mathrm{m}$ and length $l=600 \mu \mathrm{m}$ were fabricated on monolayer epitaxial graphene samples using a clean lithographic technique. ${ }^{36}$ Throughout the lithography the conducting epitaxial graphene channels were isolated from chemical residues by a protective layer of precious metal, which was removed using dilute aqua regia (DAR) at the last processing step. The DAR completely dissolves the protective precious metal layer as shown by energy-dispersive X-ray spectroscopy and X-ray photoelectron spectroscopy. While as-grown epitaxial graphene typically is heavily electron doped $\left(n \approx 10^{13} \mathrm{~cm}^{-2}\right)$, the device used in this study has low carrier concentration $n \approx$ $10^{11} \mathrm{~cm}^{-2} \cdot \cdot^{37}$ Residual molecular transfer doping brings the carrier concentration close to the Dirac point, while $n$-type carrier concentrations above $n \approx 5 \times 10^{12} \mathrm{~cm}^{-2}$ can be readily restored non-destructively ${ }^{36}$ by heating the sample at $T \geq 125^{\circ} \mathrm{C}$ in vacuum. The RG flow characteristics of several lessdisordered devices prepared similarly have been discussed in an earlier publication. ${ }^{18}$

\section{Characterization}

In graphene, low carrier concentrations are often associated with electron-hole puddles ${ }^{38,39}$ and this spatially correlated disorder leads to percolating current path $\mathrm{s}^{38}$ which convolve with slight misalignment of the voltage contacts to mix $\rho_{x x}$ with $\rho_{x y}$. In order to circumvent the mixing of resistivity components we symmetrize the resistivity ${ }^{40}$ as $\rho_{x x}(B)=\left[\rho_{x x}^{\prime}(B)+\rho_{x x}^{\prime}(-B)\right] / 2$ and $\rho_{x y}(B)=\left[\rho_{x y}^{\prime}(B)-\rho_{x y}^{\prime}(-B)\right] / 2$. The diagonal resistivity $\rho_{x x}^{\prime}$ $=\left(V_{13}+V_{1^{*} 3^{*}}\right) /\left(2 I_{\mathrm{SD}}\right)$ was measured between the two outer sets of voltage contacts $\left(V_{13}\right.$ and $\left.V_{1^{*} 3^{*}}\right)$, and the off-diagonal resistivity $\rho_{x y}^{\prime}$ was measured as the Hall resistance $R_{x y}=V_{22^{*} / I_{\mathrm{SD}}}$ across the device at the middle set of voltage contacts $\left(V_{22^{*}}\right)$ (ESI Fig. $1 \dagger$ ). All of the transport data were recorded using low-frequency lock-in measurements at source-drain current $I_{\mathrm{SD}}$ below $1 \mu \mathrm{A}$.
Magnetic field levels up to $\pm 9 \mathrm{~T}$ were applied perpendicular to the sample surface ${ }^{41,42}$ and the field was swept in the negative direction at a rate of $0.022 \mathrm{~T} \mathrm{~s}^{-1}$.

\section{Acknowledgements}

We would like to thank the Ministry of Science and Technology (MOST), Taiwan for support (grant no. MOST 105-2119-M-002048-MY3).

\section{References}

1 K. S. Novoselov, A. K. Geim, S. V. Morozov, D. Jiang, M. I. Katsnelson, I. V. Grigorieva, S. V. Dubonos and A. A. Firsov, Nature, 2005, 438, 197-200.

2 Y. Zhang, Y.-W. Tan, H. L. Stormer and P. Kim, Nature, 2005, 438, 201-204.

3 K. S. Novoselov, A. K. Geim, S. V. Morozov, D. Jiang, Y. Zhang, S. V. Dubonos, I. V. Grigorieva and A. A. Firsov, Science, 2004, 306, 666-669.

4 K. I. Bolotin, F. Ghahari, M. D. Shulman, H. L. Stormer and P. Kim, Nature, 2009, 462, 196-199.

5 X. Du, I. Skachko, F. Duerr, A. Luican and E. Y. Andrei, Nature, 2009, 462, 192-195.

6 C. R. Dean, A. F. Young, P. Cadden-Zimansky, L. Wang, H. Ren, K. Watanabe, T. Taniguchi, P. Kim, J. Hone and K. L. Shepard, Nat. Phys., 2011, 7, 693-696.

7 B. Feldman, B. Krauss, J. H. Smet and A. Yacoby, Science, 2012, 337, 1196-1199.

8 S. Bhandari, G.-H. Lee, A. Klales, K. Watanabe, T. Taniguchi, E. Heller, P. Kim and R. M. Westervelt, Nano Lett., 2016, 16, 1690-1694.

9 C. Berger, Z. Song, T. Li, X. Li, A. Y. Ogbazghi, R. Feng, Z. Dai, A. N. Marchenkov, E. H. Conrad, P. N. First and W. A. de Heer, J. Phys. Chem. B, 2004, 108, 19912-19916.

10 A. J. M. Giesbers, G. Rietveld, E. Houtzager, U. Zeitler, R. Yang, K. S. Novoselov, A. K. Geim and J. C. Maan, Appl. Phys. Lett., 2008, 93, 222109.

11 A. Tzalenchuk, S. Lara-Avila, A. Kalaboukhov, S. Paolillo, M. Syvajarvi, R. Yakimova, O. Kazakova, T. J. B. M. Janssen, V. Fal'ko and S. Kubatkin, Nat. Nanotechnol., 2010, 5, 186189.

12 T. J. B. M. Janssen, N. E. Fletcher, R. Goebel, J. M. Williams, A. Tzalenchuk, R. Yakimova, S. Lara-Avila, S. Kubatkin and V. I. Fal'ko, New J. Phys., 2011, 13, 093026.

13 A. Satrapinski, S. Novikov and N. Lebedeva, Appl. Phys. Lett., 2013, 103, 173509.

14 F. Lafont, R. Ribeiro-Palau, D. Kazazis, A. Michon, O. Couturaud, C. Consejo, T. Chassagne, M. Zielinski, M. Portail, B. Jouault, F. Schopfer and W. Poirier, Nat. Commun., 2015, 6, 6806.

15 T. J. B. M. Janssen, S. Rozhko, I. Antonov, A. Tzalenchuk, J. M. Williams, Z. Melhem, H. He, S. Lara-Avila, S. Kubatkin and R. Yakimova, 2D Mater., 2015, 2, 035015.

16 Y. Yang, C. Guangjun, P. Mende, I. G. Calizo, R. M. Feenstra, C. Chuang, C.-W. Liu, C.-I. Liu, R. J. George, A. R. H. Walker and R. E. Elmquist, Carbon, 2017, 115, 229-236. 
17 E. Pallecchi, M. Ridene, D. Kazazis, F. Lafont, F. Schopfer, W. Poirier, M. O. Goerbig, D. Mailly and A. Ouerghi, Sci. Rep., 2013, 3, 1791.

18 L.-I. Huang, Y. Yang, R. E. Elmquist, S.-T. Lo, F.-H. Liu and C.-T. Liang, RSC Adv., 2016, 6, 71977-71982.

19 C.-I. Liu, B.-Y. Wu, C. Chuang, Y.-C. Lee, Y.-J. Ho, Y. Yang, R. E. Elmquist, S.-T. Lo and C.-T. Liang, Semicond. Sci. Technol., 2016, 31, 105008.

20 M. S. Nevius, M. Conrad, F. Wang, A. Celis, M. N. Nair, A. Taleb-Ibrahimi, A. Tejeda and E. H. Conrad, Phys. Rev. Lett., 2015, 115, 136802.

21 H. P. Wei, D. C. Tsui and A. M. M. Pruisken, Phys. Rev. B: Condens. Matter Mater. Phys., 1986, 33, 1488-1490.

22 A. M. M. Pruisken, Phys. Rev. Lett., 1988, 61, 1297-1300.

23 C. P. Burgess, R. Dib and B. P. Dolan, Phys. Rev. B: Condens. Matter Mater. Phys., 2000, 62, 15359-15362.

24 Y. Xue and E. Prodan, Phys. Rev. B: Condens. Matter Mater. Phys., 2013, 87, 115141.

25 C. P. Burgess and B. P. Dolan, Phys. Rev. B: Condens. Matter Mater. Phys., 2007, 76, 113406.

26 S. H. Song, D. Shahar, D. C. Tsui, Y. H. Xie and D. Monroe, Phys. Rev. Lett., 1997, 78, 2200-2203.

27 G.-H. Kim, C.-T. Liang, C. F. Huang, J. T. Nicholls, D. A. Ritchie, P. S. Kim, C. H. Oh, J. R. Juang and Y. H. Chang, Phys. Rev. B: Condens. Matter Mater. Phys, 2004, 69, 073311.

28 M. E. Raikh and G. F. Wessels, Phys. Rev. B: Condens. Matter Mater. Phys., 1993, 47, 15609-15621.

29 S. Kivelson, D. H. Lee and S. C. Zhang, Phys. Rev. B: Condens. Matter Mater. Phys., 1992, 46, 2223-2238.

30 H. W. Jiang, C. E. Johnson, K. L. Wang and S. T. Hannahs, Phys. Rev. Lett., 1993, 71, 1439-1442.
31 T. Wang, K. P. Clark, G. F. Spencer, A. M. Mack and W. P. Kirk, Phys. Rev. Lett., 1994, 72, 709-712.

32 R. J. F. Hughes, J. T. Nicholls, J. E. F. Frost, E. H. Linfield, M. Pepper, C. J. B. Ford, D. A. Ritchie, G. A. C. Jones, E. Kogan and M. Kaveh, J. Phys.: Condens. Matter, 1994, 6, 4763-4770.

33 B. Jabakhanji, D. Kazazis, W. Desrat, A. Michon, M. Portail and B. Jouault, Phys. Rev. B: Condens. Matter Mater. Phys., 2014, 90, 035423.

34 Y. C. Lin, J. Li, S. C. de la Barrera, S. M. Eichfeld, Y. Nie, R. Addou, P. C. Mende, R. M. Wallace, K. Cho, R. M. Feenstra and J. A. Robinson, Nanoscale, 2016, 8, 8947-8954.

35 I. Forbeaux, J. M. Themlin and J. M. Debever, Phys. Rev. B: Condens. Matter Mater. Phys., 1998, 58, 16396-16406.

36 J. B. Hannon, M. Copel and R. M. Tromp, Phys. Rev. Lett., 2011, 107, 166101.

37 Y. Yang, L.-I. Huang, Y. Fukuyama, F.-H. Liu, M. A. Real, P. Barbara, C.-T. Liang, D. B. Newell and R. E. Elmquist, Small, 2015, 11, 90-95.

38 J. Martin, N. Akerman, G. Ulbricht, T. Lohmann, J. H. Smet, K. von Klitzing and A. Yacoby, Nat. Phys., 2008, 4, 144-148.

39 M. Gibertini, A. Tomadin, F. Guinea, M. I. Katsnelson and M. Polini, Phys. Rev. B: Condens. Matter Mater. Phys., 2012, 85, 201405.

40 M. Hilke, D. Shahar, S. H. Song, D. C. Tsui, Y. H. Xie and D. Monroe, Nature, 1998, 395, 675-677.

41 G. Diankov, C.-T. Liang, F. Amet, P. Gallagher, M. Lee, A. J. Bestwick, K. Tharratt, W. Coniglio, J. Jaroszynski, K. Watanabe, T. Taniguchi and D. Goldhaber-Gordon, Nat. Commun., 2016, 7, 13908.

42 C.-W. Liu, C. Chuang, Y. Yang, R. E. Elmquist, Y.-J. Ho, H.-Y. Lee and C.-T. Liang, 2D Mater., 2017, 4, 025007. 